\title{
Cerebral Metastasis Presenting after Complete Primary Resection of Atrial Myxoma: Case Report
}

\author{
Ian Côté, John Sinclair, John Woulfe, Rafael Glikstein, John Veinot
}

Keywords: Brain tumours, Cardiology, Neuro-oncology - surgical, Neurosurgery

doi:10.1017/cjn.2015.293

Can J Neurol Sci. 2015; 42: 457-460

Cardiac tumours most frequently occur following metastatic spread from other primary source but primary lesions do occur, with the most frequent being left atrial myxoma. While the histopathology of this mesenchymal lesion is generally benign, the location of most myxomas in the left atrium potentiates systemic embolization as a frequent mechanism of tumor spread and clouds an understanding about the malignant potential of these deposits. Neurologic and systemic embolization of atrial myxoma has been well documented and may occur in as much as half of patients, with brain lesions often noted before the diagnosis of intracardiac tumor but also noted as soon as two months, and as long as eight years, after initial resection after the initial complete resection. ${ }^{1,2}$

Neurological complications of embolization from left atrial myxomas may take various forms. The first is formed brain metastases with consequent raised intracranial pressure, neurological deficits, or seizures. Alternately, arterial occlusion by the embolic particles may cause vertebral infarction or haemorrhage with similar consequence. Lastly, aneurysmal formations in the major cerebral arteries may occur, most frequently in the middle carotid artery territory.

We present a patient treated for a benign atrial myxoma two years prior to the presentation of multiple intracranial parenchymal metastasis and, remarkably, in the absence of local recurrence.

\section{Case Report}

A 46 year old right handed woman presented with a three month history of seizure activity manifesting as paresthesias involving her left lower abdomen and upper thigh. She was asymptomatic interictally, had no complaints of headache or of nausea or vomiting, of weakness or sensory deficits, nor of visual or auditory disturbances.

Past medical history of significance included complete resection of an inter-atrial septal cardiac myxoma two years previously. Social history reveals a non-smoker, non-drinker, non-intravenous drug user with no recent travel history.

Physical examination revealed a well appearing, alert and oriented patient. Normal cardiovascular and peripheral vascular exams were observed. Neurological exam revealed grade 4 weakness of the left hip flexor, reduced light touch and pinprick sensation involving the left lower thoracic/abdominal/thigh areas, accentuated left knee jerk reflex and equivocal left plantar reflex. No papilledema was observed.

\section{Imaging}

Non-contrast computed tomography scan of the head revealed three hyperdense lesions, surrounded by vasogenic oedema, interpreted as hemorrhagic (Figure 1). Magnetic resonance imaging (MRI) without and with gadolinium confirmed the presence of three hypointense lesions on T1 and gradient echo sequences. They were mildly enhancing, surrounded by vasogenic edema and located at the gray-white matter junction in both cerebral hemispheres (Figure 2). The largest lesion was located at the level of the right central sulcus measuring $11.3 \mathrm{~mm}$. The other two lesions were identified in the right inferior frontal gyrus and left precentral gyrus, measuring 8.3 and $8.8 \mathrm{~mm}$ respectively. Cerebral angiography did not reveal any aneurismal lesions. Cardiac MRI investigations with axial HASTES and true FISP of the chest, axial CINES of the heart and four chamber CINES of the heart were done and demonstrated the absence of any intracardiac recurrence of the atrial myxoma. Magnetic resonance imaging of the spine did not show any further lesions.

The patient underwent three procedures. The first intervention was a diagnostic open biopsy of the right frontal lesion yielding a diagnosis of metastatic myxoma. Valproic acid was started preoperatively. The second intervention was an awake craniotomy with cortical and subcortical mapping to resect the right motor cortex lesions. The third intervention was a craniotomy under general anaesthesia to respect the left-sided lesion. Following these operations, the patient's left hip flexor weakness improved gradually and she was discharged to a short term rehabilitation program. Three month follow up MRI of the brain showed decreased vasogenic oedema but increased size of the right motor cortex cystic lesion which remains neurologically silent. Treatment with chemotherapy or radiotherapy was not pursued. The anticonvulsant regiment was continued for one year and was eventually stopped with no recurrent seizure activity.

\section{Histology}

The atrial myxoma originally resected had free margins and with benign features such as a stellate or pseudovascular pattern,

From the Department of Neurosurgery (IC), University of Miami MILLER School of Medicine, Miami, Florida, USA; Department of Surgery (JS), Division of Neurosurgery, Department of Pathology; Laboratory Medicine (JW); Department of Radiology (RG), The Ottawa Hospital Civic Campus; Department of Pathology and Laboratory Medicine (JV), University of Ottawa Heart Institute, Ottawa, Ontario, Canada.

Received January 20, 2015. Final Revisions Submitted April 22, 2015. Correspondence to: Ian Côté, 1095 NW $14^{\text {th }}$ Terrace, Miami, Florida, USA, 33125. Email: ixc118@med.miami.edu 
degenerative changes with organizing fibrin, areas with hemosiderin laden macrophages and fibrosis. The specimen obtained from the incisional biopsy was a brown, bilobed, well circumscribed structure. The intraoperative frozen section favoured a vasculitic process but presented small myxoid areas unexplained by this preliminary diagnosis. Permanent sections confirmed

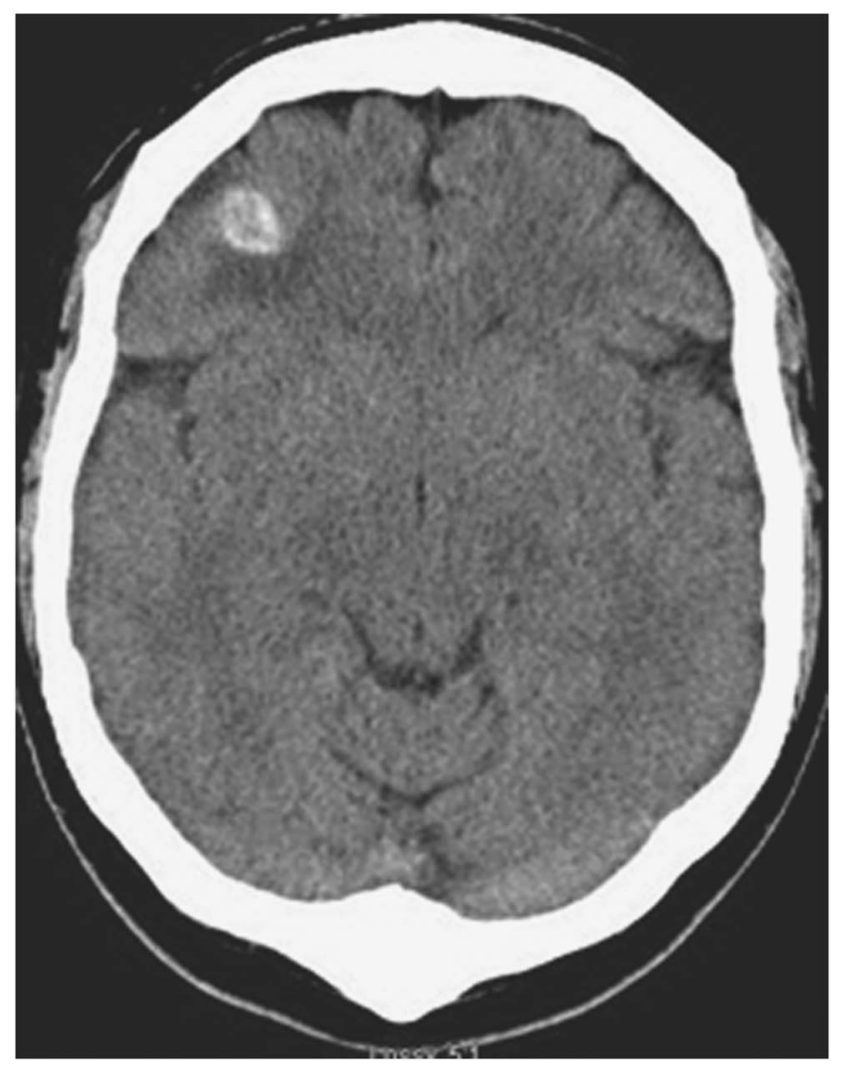

Figure 1: Non enhanced axial computed tomography of the head showing a right well-circumscribed haemorrhagic frontal lesion and surrounding vasogenic oedema. the presence of metastatic myxomatous disease with identical features. Gamma-Gandy fibers within multinucleated giant cells were observed. No malignant features were appreciated such as mitotic figures or necrosis. The vessels in which the embolus originally lodged were no longer identifiable. Immunohistochemically, the constituent neoplastic cells showed staining for CD34, CD31, and vimentin. They were negative for smooth muscle actin, desmin, and S100 (Figure 3).

\section{DisCUSSION}

Primary cardiac tumours are rare diseases. In large autopsy series, they are reported to have an incidence between 0.0017 and $0.28 \%$. Three quarters of primary cardiac tumours are benign, of which nearly half are myxomas. In up to $90 \%$ of cases, myxomas arise from the interatrial septum and expand into the left atrium. They occur more frequently in women between the third and sixth decades of life. Myxomas usually present as embolic manifestations, cardiac failure or constitutional symptoms such as fever, anemia, weight loss, malaise accompanied by a raised erythrocyte sedimentation rate ${ }^{1}$.

There are two main theories as to the histogenesis of myxomas. In the first, Krikler et al. ${ }^{3}$ demonstrated that a significant proportion of myxomas express Schwann cell and neuroendocrine differentiation markers such as protein gene product (PGP), neurone specific enolase (NSE) and synaptophysin. This suggests that myxomas originate from endocardial sensory nerve tissue. In the second theory, Pucci et al. ${ }^{4}$ demonstrated that myxomatous stromal cells are reactive for neuropeptides and endothelial markers suggesting that myxomas arise from multipotent mesenchymal cells capable of both neural and endothelial differentiation. Additionally, they detected glands in certain myxomas showing immunoreactivity pattern overlapping that of endocrine cells from normal human gut thus supporting the hypothesis that they represent rests of entrapped embryonic foregut.

Systemic embolization can occur in 6 to $43 \%$ of patients, of which half affect the head and neck vessels ${ }^{1,5}$. In Ekinci et al.'s
A

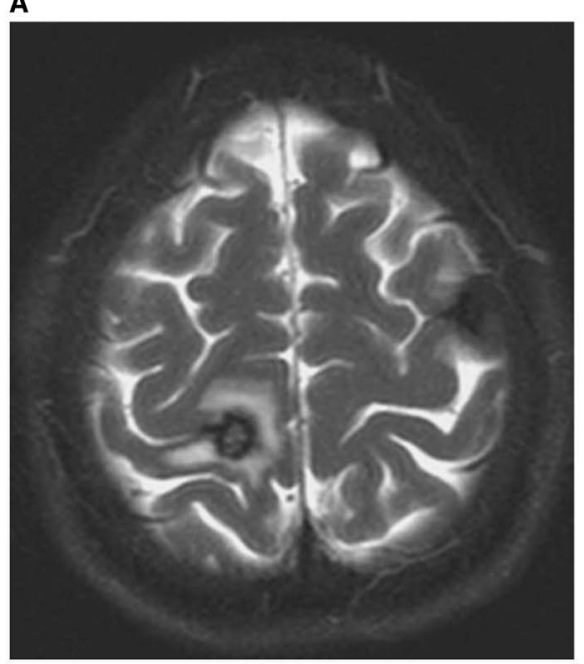

B

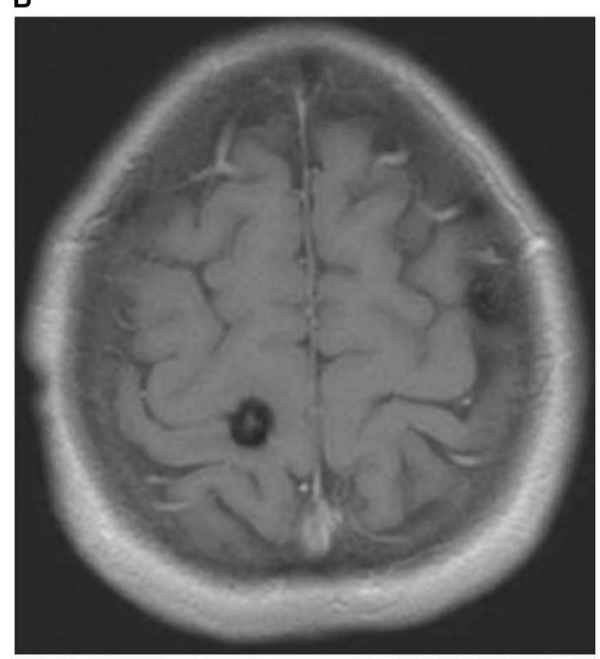

Figure 2: Magnetic resonance imaging of the brain; a) hypointense T2 signal lesions with vasogenic oedema on right and left frontal lobes; $b$ ) minimal degree of enhancement on bilateral frontal T1 signal lesions. 
A

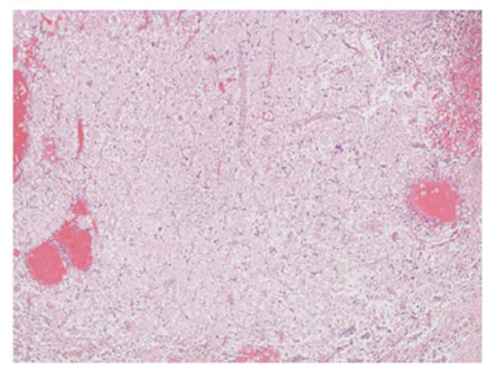

B

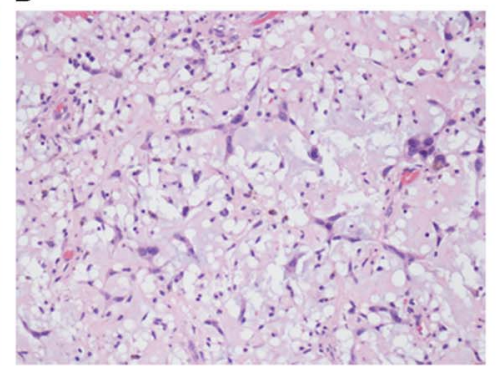

C

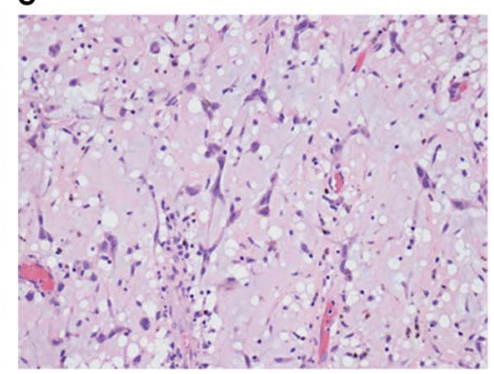

Figure 3: Histopathology of right frontal lesion $(A, B)$ and original atrial myxoma $(C)$. Hemotoxylin and eosin staining. A) $4 x$ magnification showing a paucicellular, markedly myxoid lesion. B) $20 x$ magnification showing spindle cells disposed randomly and in perivascular and pseudovascular patterns on a myxoid background. Also present were hemosiderin-laden macrophages, Gamma-Gandy fibres, and multicucleated giant cells. C) $20 x$ magnification of the original atrial myxoma showing histological features identical to those in " $b$ ".

series of 113 patients $^{6}$, the most common neurologic presentation was ischemic stroke ( $82 \%$ ) followed by syncope $(28 \%)$, psychiatric presentation (23\%), headache (13\%), seizures (12\%) and intracerebral haemorrhage (12\%). At the time of diagnosis, $11 \%$ had myxomatous cerebral aneurysms.

Development of cerebral metastasis from benign myxoma following complete curative local resection is only rarely described and the mechanism by which a histologically benign myxoma can have malignant potential has yet to be elucidated. Kanda et al. ${ }^{5}$ have suggested that cardiac myxoma cells produce interleukin-6, which may play a crucial role in the adhesiveness of myxoma cells to cerebral microvessel endothelial cells. These myxoma cells may then migrate transendothelially and develop metastasis. Some cases have described the disruption of the arterial internal elastic lamina by embolic tumor ${ }^{1}$. Another study showed overproduction of CXC chemokines (a sub-group of small chemokine proteins), interleukin-8 and growth related oncogene by myxoma cells which may partly explain their metastatic potential ${ }^{7}$.

The histological characteristics of the cerebral metastatic myxoma presented in this case are similar to those described previously. The vessel in which the embolus originally lodged itself was no longer identifiable, a trait that differs from previous descriptions. The presence of robust inflammatory changes and vasculitic changes associated with the lesion suggests a strong focal inflammatory response. This contributes to the initial difficulty in diagnosis during intra-operative frozen section and the inclusion of generalized vasculitis in the differential diagnosis.

The treatment of choice for cardiac myxomas is surgical removal and should be performed immediately to reduce embolic phenomena. It is usually curative. 20-year survival rate of $85 \%$ and local recurrence rates as low as $4.7 \%$ have been reported ${ }^{8}$. The benign nature of these tumors, which exhibit cells without mitotic figures, precludes the use of radiation therapy or chemotherapy as adjunctive measures of treatment.

There is currently no standard management for cerebral embolic myxoma. Previously described cases have approached this problem utilizing surgery with or without the use of adjuvant radiotherapy or chemotherapy. In Altundag et al.'s review of literature ${ }^{9}$, various authors opted for surgery alone for treatment as with our case. Surgery was performed in cases having one or two metastatic lesions only. Of those cases, three were alive with the disease at 18 to 120 months follow up, one died of the disease at 53 months follow up, two were alive with no evidence of disease at 9 and 21 months follow up and one was lost to follow up. Bernet et al. $^{2}$ described a patient who had multiple bilateral cerebral metastasis and two soft tissue metastasis presenting two months after cardiac surgery. All three treatment modalities were utilized and the patient demonstrated complete remission ten years following initial resection. In our case, three separate operative approaches where utilized. Follow up imaging has shown recurrence of one lesion in an eloquent portion of brain although it is currently neurologically silent.

\section{ConClusion}

Myxomas can rarely cause metastatic disease to the brain. Histological features suggest these tumors behave in a benign manner. In this case operative management was chosen to address the patient's seizure disorder. Resection of up to three metastatic cerebral myxomas was attempted and was deemed a viable option as harm could be minimized. Adjunctive radiotherapy or chemotherapy could not be supported as no malignant histological features were observed. Cardiac surgery literature supports primary resection as the treatment of choice. Observation of clinically silent recurrent lesions is appropriate as no strong evidence to treat exists. Considering the benign nature of this disease, we recommend a conservative approach to monitoring with initial brain MRI and cardiac imaging at three months, followed by yearly scans for two years, then biennially for four years for a total of 10 years follow up.

\section{Disclosures}

Ian Côté, John Sinclair, Rafael Glikstein and John Veinot have nothing to disclose. John Woulfe has the following disclosures: Michael J. Fox Foundation, Researcher, Research grant; Pathology and Laboratory Medicine, University of Ottowa, Researcher, Research grant; CIHR, Researcher, Research grant

\section{REFERENCES}

1. DeSousa AL, Muller J, Campbell RL, Batnitsky S, Rankin L. Atrial myxoma: a review of the neurological complications, metastases, and recurrences. J Neurol Neurosurg Psychiatry. 1978;41:1119-24. 
2. Bernet F, Sulz PM, Carrel TP. Long-term remission after resection, chemotherapy, and irradiation of metastatic myxoma. Ann Thorac Surg. 1998;66:1791-2.

3. Krikler DM, Rode J, Davies MJ, Woolf N, Moss E. Atrial myxoma: a tumour in search of its origins. Brit Heart J. 1992;67:89-91.

4. Pucci A, Galiardotto P, Zanini C, et al. Histopathologic and clinical characterization of cardiac myxoma: Review of 53 cases from a single institution. Am Heart J. 2000;140:134-8.

5. Kanda T, Sakamaki T, Murate K. A cardiac myxoma with interleukin-6 production and cerebral metastasis. Int J Cardiol. 1994;45:144-6.
6. Ekinci EI, Donnan GA. Neurological manifestations of cardiac myxoma: a review of the literature and report of cases. Int Med J. 2004;34:243-9.

7. Sakamoto H, Sakamaki T, Sawada Y, et al. Production of endothelin1 and big endothelin-1 by human cardiac myxoma cells implication of the origin of myxomas. Circ J. 2004;68:1230-2.

8. Bjessmo S, Ivert T. Cardiac myxoma: 40 years' experience in 63 patients. Ann Thorac Surg. 1997;63:697-700.

9. Altundag MB, Ertas G, Durmus S, et al. Brain metastasis of cardiac myxoma: case report and review of the literature. J Neuro-Oncol. 2005;75:181-4 